\title{
Influence of surface anisotropy on exchange resonance modes in spherical shells
}

\author{
C McKeever ${ }^{1, *}$, F Y Ogrin ${ }^{1}$ andMM Aziz ${ }^{2}$ \\ ${ }^{1}$ Department of Physics and Astronomy, University of Exeter, Exeter, EX4 4QL, United Kingdom \\ ${ }^{2}$ Department of Engineering, University of Exeter, Exeter, EX4 4QF, United Kingdom
}

\begin{abstract}
The dynamical properties of saturated spherical shells are investigated in the exchange-dominated regime when assuming that surface anisotropy is present at both the inner and outer boundaries. It is found that surface anisotropy plays an important role in determining the dependence of lower-ordereigenvalueson shell thickness. The mode frequency can increase with decreasing shell thickness, oris driven rapidly towards the ferromagnetic resonance frequency depending on the choice of the surfaceanisotropy constant $K_{S}$ at each boundary. The presence of surface anisotropy significantlymodifies the size dependence of the modes which can be suppressed or amplified based on the coupling between boundaries. Similar size dependent behaviour to the solid sphere is observedfor lower-order eigenvalues in the presence of surface anisotropy up to a thickness of $R_{1} / R_{2} \sim 0.5$ after which large deviations begin to occur, where $R_{1}$ and $R_{2}$ are the inner and outer radius, respectively. Moreover, surface anisotropyintroduces a dependence of the zeroth mode on shell thickness, removing the degeneracy with the ferromagnetic resonance and leading to apronounced size dependence of this modefor thin shells.
\end{abstract}

Keywords:Surface Anisotropy Nanoshell Exchange Resonance

\section{Introduction}

Nano- and microscale ferromagnetic particles have been a source of intense research interest due their novel fundamental properties and broad range of applications, such as magnetic memory storage[1][2][3], microwave oscillators [4][5] and cancer treatment [6][7].The static and dynamic properties of curved geometrical structures such as hemispheres, nanotubes and spherical shells have undergone an explosion of interest in recent years, as part of a broader trend towards three-dimensional nanomagnetism[8]. In particular, advancements in chemical synthesis methods have enabled the production of nanosized hollow spheres with narrow size distributions and diameters ranging from tens to hundreds of nanometers[9][10][11][12][13][14][15]. There has been great interest in how surface anisotropy will alter the fundamental magnetic properties of nanostructures, which may emerge due to embedding magnetic particles in non-magnetic matrices [16][17], crystallographic arrangement on the surface [18], expansion and contraction of the lattice structure [19], among numerous other physical and chemical effects [20][21]. As a consequence, surface magnetism of nanoparticles has been the subject of rigorous experimental [22][23][24] and theoretical [25][26][27][28] investigation. For example, surface anisotropy may be responsible for the high perpendicular magnetic anisotropy observed experimentally in thin epitaxial films of hcp cobalt 
[29][30][31] and other multi-layered ferromagnetic materials [32][33][34]. When the surface anisotropy constant $K_{S}$ is negative and of sufficiently large absolute value, the magnetization vector can be orientated perpendicular to the film surface despite the presence of large demagnetizing fields, a phenomenon which is potentially useful for perpendicular magnetic recording.

Gaididei et al derived a magnetic energy functional for an arbitrary smoothly curved thin shellunder the assumption that the magnetostatic effects can be reduced to an effective easysurface anisotropy[35]. It was shown that the curvilinear geometry brings about an effective exchange-driven anisotropy and an effective Dzyaloshinskii-Moriya interaction (DMI). As a consequence, magnetic skyrmions can be stabilized on a spherical shell by curvature effects only, even when the intrinsic DMI is absent [36]. The emergent curvature-induced anisotropy and an effective DMI leads to polarity-chirality coupling [37], increased domain wall velocities [38] and curvature-driven magnetochirality[39]. Magnetochiral effects have been demonstrated in studies of the Möbius ring [40] and torus [41]. Moreover, it has been shown [42][43] that toroidal nanomagnets can stabilize a magnetic vortex down to smaller sizes than has been previously reported in their cylindrical counterparts[44][45].

Surface anisotropy may also play an important role in the size-dependent dynamical properties of ferromagnetic nanospheres[46][47][48]. The dynamic permeability measurements of these spherical particles exhibit several narrow resonance bands which have been attributed to exchange resonance modes[49]. These modes have been a source of great research interest due to their negligible eddy current loss [50] andhave been adopted in the analysis of a wide range of material composites[51][52][53][54][55]in order toextract the magnetic parameters and estimate surface contributions to the resonance frequency.The formula for exchange resonance modes was first derived [56] by neglecting the magnetostatic contribution to the resonance, resulting in resonance frequencies which possess a $1 / R_{2}^{2}$ dependence on the particle size, where $R_{2}$ is the outer radius of the sphere. This approximation is justified for sufficiently small particles when the exchange energy dominates over the magnetostatic energy, in contrast to the magnetostatic approximation in large particles, for which the exchange term is neglected [57].

Recently, the microwave properties of core-shell and magnetically hollow particles have been the subject of considerableinterest[58][59][60][61][62][63]and the size dependent permeability of hollownickel[64]and carbonyl iron[65]particleshas been measured.Acore-shell or multilayered particle offers tuneable electromagnetic properties, lighter weight and awidefrequency bandwidth at the cost of increased sensitivity of the ferromagnetic shell to surface imperfections. However,little is understood about the influence of surface anisotropy on the high frequency performance of such nanoparticles, whereprevious theoretical and experimental treatment has focused on solid spheres. Here,the exchange resonance theory is generalizedwithin a rigorous micromagnetic framework in order to study the effect of surface anisotropy on the resonance frequency for different values of shell thickness and size.This can provide detailed understanding into the high frequency dynamics and improve accuracy when fittingmeasured permeability spectra to theoretical resonance curves.

\section{Theory}

Néel proposed a phenomenological model of the magnetic surface anisotropy [66] to account for the breaking of crystallographic symmetry at the particle surface. Macroscopic expressions for the surface anisotropy energy density were later suggested by Brown [67] and Aharoni[68]. Here, we consider a uniaxial anisotropy density $w_{s}$ of the form 


$$
w_{s}=K_{s}\left(1-m_{z}^{2}\right)
$$

where $m_{z}$ is the z-component of the magnetisation. If the magnetisation $\mathbf{m}$ is assumed to be parallel to zbefore nucleation, the micromagnetic boundary conditions of (1) are given by the equations [67]

$$
\begin{aligned}
& \frac{\partial m_{x}}{\partial n}+\frac{2 K_{s}}{C} m_{x}=0 \\
& \frac{\partial m_{y}}{\partial n}+\frac{2 K_{s}}{C} m_{y}=0
\end{aligned}
$$

where $C$ is the exchange constant, $K_{s}$ is the anisotropy constant and $n$ is normal to the surface, which for a spherical particle is given by the spherical coordinate $r$. The linearized differential equations for the exchange modes are given by [68]

$$
\left(\nabla^{2}-\frac{M_{s} H_{z}}{C}\right) m_{y}+\frac{M_{s}}{\gamma_{0} C} \frac{\partial m_{x}}{\partial t}=0
$$

and

$$
\left(\nabla^{2}-\frac{M_{s} H_{z}}{C}\right) m_{x}-\frac{M_{s}}{\gamma_{0} C} \frac{\partial m_{y}}{\partial t}=0
$$

where $M_{S}$ is the saturation magnetisation and $C=2 A$ is the exchange constant.Assuming that a sufficiently large DC field is present to saturate the particle, then the expression $H_{z}$ is given, for the case of a solid sphere, by

$$
H_{z}=H_{0}+\frac{2 K_{1}}{M_{S}}
$$

where $H_{0}$ is the external DC field applied parallel to an anisotropy easy axis, $K_{1}$ is the anisotropy constant for either uniaxial or cubic volume anisotropy, $\gamma_{0}$ is the gyromagnetic ratio, $t$ is time, and $\boldsymbol{m}=\boldsymbol{M} /|\boldsymbol{M}|$ is a unit vector parallel to the magnetization vector $\boldsymbol{M}$. The boundary conditionsfor each surface, in the presence of surface anisotropy, are given by

$$
\begin{gathered}
\left(\frac{\partial m_{x}}{\partial r}+\frac{2 K_{S_{1}}}{C} m_{x}\right)_{r=R_{1}}=\left(\frac{\partial m_{x}}{\partial r}+\frac{2 K_{S_{2}}}{C} m_{x}\right)_{r=R_{2}} \\
=\left(\frac{\partial m_{y}}{\partial r}+\frac{2 K_{S_{1}}}{C} m_{y}\right)_{r=R_{1}}=\left(\frac{\partial m_{y}}{\partial r}+\frac{2 K_{S_{2}}}{C} m_{y}\right)_{r=R_{2}}=0(6)
\end{gathered}
$$

Here, two surface anisotropy constants $K_{s_{1}}$ and $K_{s_{2}}$ are introduced, corresponding to the inner and outer boundaries, respectively. The general solution of equations (4) and (5) can be obtained by separation of the variables in terms of the spherical coordinates $r, \theta$ and $\varphi$, given by 


$$
m_{x}=e^{i \omega t} e^{i s \theta} P_{n}^{s}(\cos \theta)\left(A_{1} j_{n}\left(\frac{\mu r}{R_{2}}\right)+A_{2} y_{n}\left(\frac{\mu r}{R_{2}}\right)\right)
$$

and

$$
m_{y}=e^{i \omega t} e^{i s \theta} P_{n}^{s}(\cos \theta)\left(B_{1} j_{n}\left(\frac{\mu r}{R_{2}}\right)+B_{2} y_{n}\left(\frac{\mu r}{R_{2}}\right)\right)
$$

where $A, B, \omega$ and $\mu$ are real constants, $\mathrm{s}$ and $n \geq s$ are integers, $P_{n}^{s}$ is the Legendre function and $j_{n}$ and $y_{n}$ are the spherical Bessel functions of the first and second kind, respectively. Substituting equations (7) and (8) into equations (4) and (5) gives,

$$
\left(\frac{\mu^{2}}{R_{2}^{2}}+\frac{M_{S} H_{z}}{C}\right) A_{j}+\frac{i \omega M_{s}}{\gamma_{0} C} B_{j}=\frac{i \omega M_{s}}{\gamma_{0} C} A_{j}-\left(\frac{\mu^{2}}{R_{2}^{2}}+\frac{M_{s} H_{z}}{C}\right) B_{j}=0
$$

for $j=1$ and 2. The determinant of the coefficients of $A_{j}$ and $B_{j}$ must be zero if (9) has a common, nonzero solution. Equating the determinants to zero gives,

$$
\left(M_{s} H_{z} / C+\mu^{2} / R_{2}^{2}\right)^{2}=\left(\omega M_{s} / \gamma_{0} C\right)^{2}
$$

The resonance frequencies $\omega$ are then given by,

$$
\omega= \pm \gamma_{0}\left(C \mu^{2} /\left(R_{2}^{2} M_{S}\right)+H_{z}\right)
$$

Now, it is only necessary to fulfil the boundary conditions (6). At first there are four equations to solve, however the problem can be simplified by noting that the substitution of (10) into (9) gives,

$$
i B_{1} \pm A_{1}=0 \text { and } i B_{2} \pm A_{2}=0
$$

The terms to be substituted into the boundary conditions (6) can be calculated from the expressions (2) and (3), namely

$$
\begin{gathered}
\frac{\partial m_{x}}{\partial r}+\frac{2 K_{s}}{C} m_{x}= \\
e^{i w t} e^{i s \theta} P_{n}^{s} \cos (\theta)\left(\frac{\mu}{R_{2}} A_{1} \frac{\partial j_{n}\left(\frac{\mu r}{R_{2}}\right)}{\partial\left(\frac{\mu r}{R_{2}}\right)}+\frac{\mu}{R_{2}} A_{2} \frac{\partial y_{n}\left(\frac{\mu r}{R_{2}}\right)}{\partial\left(\frac{\mu r}{R_{2}}\right)}+\frac{2 K_{s}}{C} A_{1} j_{n}\left(\frac{\mu r}{R_{2}}\right)+\frac{2 K_{s}}{C} A_{2} y_{n}\left(\frac{\mu r}{R_{2}}\right)\right)
\end{gathered}
$$




$$
\begin{gathered}
\frac{\partial m_{y}}{\partial r}+\frac{2 K_{s}}{C} m_{y}= \\
e^{i w t} e^{i s \theta} P_{n}^{s} \cos (\theta)\left(\frac{\mu}{R_{2}} B_{1} \frac{\partial j_{n}\left(\frac{\mu r}{R_{2}}\right)}{\partial\left(\frac{\mu r}{R_{2}}\right)}+\frac{\mu}{R_{2}} B_{2} \frac{\partial y_{n}\left(\frac{\mu r}{R_{2}}\right)}{\partial\left(\frac{\mu r}{R_{2}}\right)}+\frac{2 K_{s}}{C} B_{1} j_{n}\left(\frac{\mu r}{R_{2}}\right)+\frac{2 K_{s}}{C} B_{2} y_{n}\left(\frac{\mu r}{R_{2}}\right)\right)
\end{gathered}
$$

Substituting equations (12) and (13) into (6) and using the relations (11) to substitute for $B_{1}$ and $B_{2}$, it is readily seen that to fulfil all boundary conditions, it is necessary and sufficient to fulfil only

$$
\begin{aligned}
& e^{i \omega t} e^{i s \theta} P_{n}^{s}(\cos \theta)\left(\frac{\mu}{R_{2}} A_{1} \frac{\partial j_{n}(\gamma)}{\partial \gamma}+\frac{\mu}{R_{2}} A_{2} \frac{\partial y_{n}(\gamma)}{\partial \gamma}+\frac{2 K_{S_{1}}}{C} A_{1} j_{n}(\gamma)+\frac{2 K_{S_{1}}}{C} A_{2} y_{n}(\gamma)\right)_{\gamma=\frac{\mu R_{1}}{R_{2}}} \\
& =e^{i \omega t} e^{i s \theta} P_{n}^{s}(\cos \theta)\left(\frac{\mu}{R_{2}} A_{1} \frac{\partial j_{n}(\mu)}{\partial \mu}+\frac{\mu}{R_{2}} A_{2} \frac{\partial y_{n}(\mu)}{\partial \mu}+\frac{2 K_{S_{2}}}{C} A_{1} j_{n}(\mu)+\frac{2 K_{S_{2}}}{C} A_{2} y_{n}(\mu)\right)=0
\end{aligned}
$$

where we have defined $\gamma=\mu R_{1} / R_{2}$. By cancelling the $e^{i w t} e^{i s \theta} P_{n}^{s}(\cos \theta)$ term and equating $A_{1}$ and $A_{2}$, these expressions can be re-written as

$$
\begin{aligned}
A_{1}\left(\frac{\mu}{R_{2}} \frac{\partial j_{n}(\gamma)}{\partial \gamma}\right. & \left.-\frac{2 K_{S_{1}}}{C} j_{n}(\gamma)\right)_{\gamma=\frac{\mu R_{1}}{R_{2}}}+A_{2}\left(\frac{\mu}{R_{2}} \frac{\partial y_{n}(\gamma)}{\partial \gamma}-\frac{2 K_{S_{1}}}{C} y_{n}(\gamma)\right)_{\gamma=\frac{\mu R_{1}}{R_{2}}} \\
& =A_{1}\left(\frac{\mu}{R_{2}} \frac{\partial j_{n}(\mu)}{\partial \mu}+\frac{2 K_{S_{2}}}{C} j_{n}(\mu)\right)+A_{2}\left(\frac{\mu}{R_{2}} \frac{\partial y_{n}(\mu)}{\partial \mu}+\frac{2 K_{S_{2}}}{C} y_{n}(\mu)\right)=0
\end{aligned}
$$

Suchapairofequationshasnon-zerosolution for $A_{1}$ and $A_{2}$ providedthedeterminantoftheircoefficients vanishes, leaving

$$
\begin{aligned}
\left(\frac{\mu}{R_{2}} \frac{\partial j_{n}(\mu)}{\partial \mu}+\frac{2 K_{S_{2}}}{C} j_{n}(\mu)\right)\left(\frac{\mu}{R_{2}} \frac{\partial y_{n}(\gamma)}{\partial \gamma}-\frac{2 K_{S_{1}}}{C} y_{n}(\gamma)\right)_{\gamma=\frac{\mu R_{1}}{R_{2}}} \\
-\left(\frac{\mu}{R_{2}} \frac{\partial y_{n}(\mu)}{\partial \mu}+\frac{2 K_{S_{2}}}{C} y_{n}(\mu)\right)\left(\frac{\mu}{R_{2}} \frac{\partial j_{n}(\gamma)}{\partial \gamma}-\frac{2 K_{S_{1}}}{C} j_{n}(\gamma)\right)_{\gamma=\frac{\mu R_{1}}{R_{2}}}=0
\end{aligned}
$$

The eigenvalues $\mu_{k n}$ can be calculated from the transcendental equation (14) for different values of the outer radius $R_{2}$ and ratio $R_{1} / R_{2}$. For the case that $K_{S_{2}}=K_{S_{1}}=0$, when no surface anisotropy is present at either boundary, the expression (14) reduces to 


$$
\left(\frac{\partial j_{n}(\mu)}{\partial \mu}\right)\left(\frac{\partial y_{n}(\gamma)}{\partial \gamma}\right)_{\gamma=\frac{\mu R_{1}}{R_{2}}}-\left(\frac{\partial y_{n}(\mu)}{\partial \mu}\right)\left(\frac{\partial j_{n}(\gamma)}{\partial \gamma}\right)_{\gamma=\frac{\mu R_{1}}{R_{2}}}=0
$$

which is the expression for the eigenvalues of the exchange resonance modes in a hollow ferromagnetic sphere when surface anisotropy is not present. It is readily seen that equation (14) introduces a dependence of the eigenvalues on the outer radius $R_{2}$, in addition to the ratio $R_{1} / R_{2}$.

For an ideally saturated solid sphere, the demagnetizing factor along the $z$-axis does not feature in the expression for the ferromagnetic resonance $H_{z}[70]$. Here, the demagnetizing factors are equal in all directions and have no overall contribution to the exchange resonance frequency. However, the demagnetizing field is inhomogeneous for the hollow sphere, and it is necessary to consider the demagnetizing factor when calculating the ferromagnetic resonance in this situation. Recently, Prat-Camps et al calculated exact analytical expressions for the volume $\left(N_{m}\right)$ and mid-plane $\left(N_{f}\right)$ averaged demagnetizing factors of the hollow sphere [71] which are expressed in terms of the static magnetic susceptibility of the particle,

$$
N_{m}=\frac{1}{3}-\frac{2 p^{3} \chi}{6 \chi+9}
$$

and

$$
N_{f}=\frac{1}{3}-\frac{2 p^{2}\left(3+\chi\left(1+p+p^{2}\right)\right)}{9(1+p)+6 \chi\left(1+p+p^{2}\right)}
$$

where $\chi$ is the static magnetic susceptibility and $p=R_{1} / R_{2}$. The change in the demagnetizing factor is small for $R_{1} / R_{2} \leq 0.2$ but grows rapidly with increasing $R_{1} / R_{2}$ and becomes large for thin shells.An ideally saturated shell has only non-zero $N_{z}$ components when the applied field is directed along the $z$-axis, while for more complex magnetisation configurations[72]it maybe necessary toconsider variations in $N_{x}$ and $N_{y}$.

\section{Results and Discussion}

\section{Shell thickness}


(a)

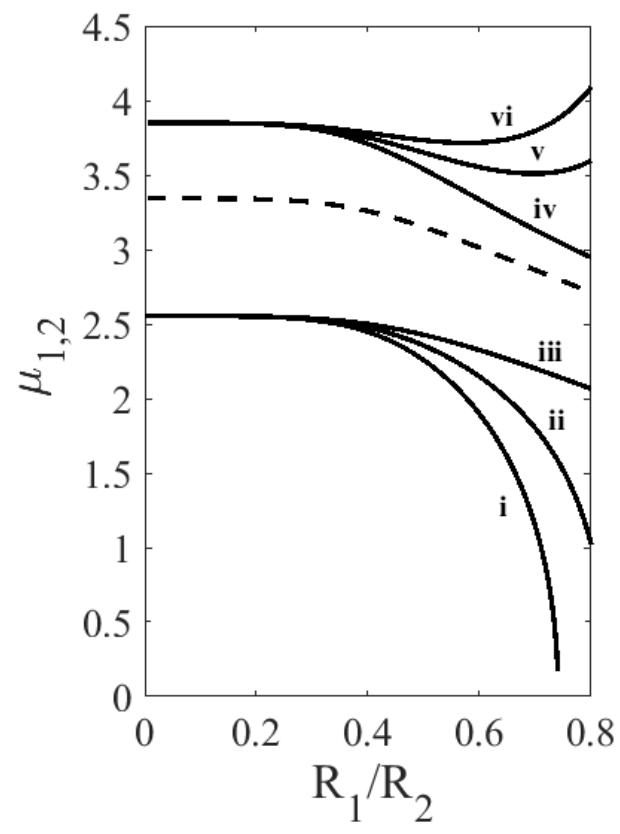

(b)

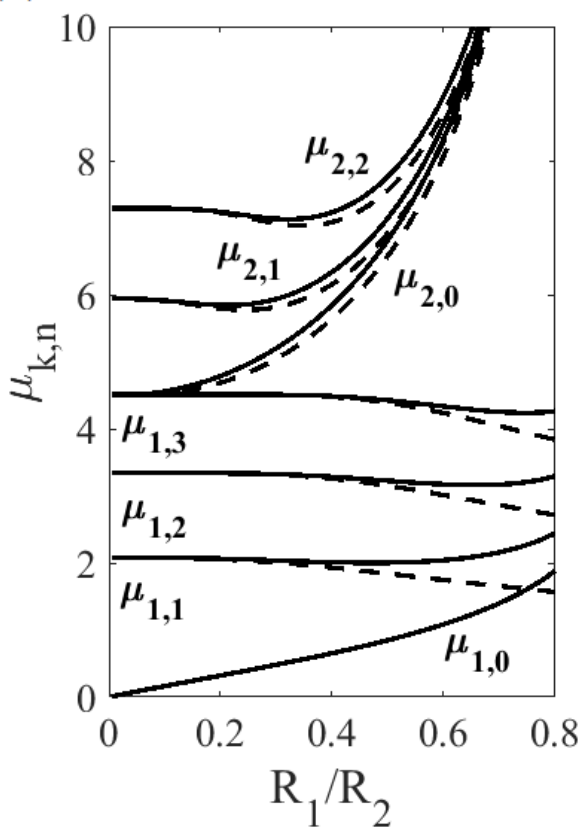

Figure 1: (a) $\mu_{1,2}$ plotted against the ratio $R_{1} / R_{2}$ for the condition $K_{S_{2}}=-4 \times 10^{-4} \mathrm{~J} / \mathrm{m}^{2}$ with (i) $K_{s_{1}}=-4 \times 10^{-4} \mathrm{~J} / \mathrm{m}^{2}$, (ii) $K_{s_{1}}=0 \mathrm{~J} / \mathrm{m}^{2}$ and(iii) $K_{s_{1}}=4 \times 10^{-4} \mathrm{~J} / \mathrm{m}^{2}$ and $K_{s_{2}}=4 \times$ $10^{-4} \mathrm{~J} / \mathrm{m}^{2}$ with (iv) $K_{S_{1}}=-4 \times 10^{-4} \mathrm{~J} / \mathrm{m}^{2}$, (v) $K_{S_{1}}=0 \mathrm{~J} / \mathrm{m}^{2}$ and(vi) $K_{S_{1}}=4 \times 10^{-4} \mathrm{~J} /$ $\mathrm{m}^{2}(\mathrm{~b}) \mu_{k, n}$ plotted against the ratio $R_{1} / R_{2}$ for $K_{S_{2}}=0, K_{S_{1}}=4 \times 10^{-4} \mathrm{~J} / \mathrm{m}^{2} . I n$ all cases the dashed line represents $K_{s_{2}}=K_{s_{1}}=0$.

Here, we consider the case of iron particles with exchange constant $2.1 \times 10^{-11} \mathrm{~J} / \mathrm{m}$ and outer radius $R_{2}=50 \mathrm{~nm}$. In thin films, absolute values of $K_{s}$ have been found in the range $K_{s}=$ $0.6-4.5 \times 10^{-4} \mathrm{~J} / \mathrm{m}^{2}$ for FePt films [73] and $K_{s}=1.7-9.6 \times 10^{-4} \mathrm{~J} / \mathrm{m}^{2}$ for different interfaces of iron at room temperature [74]. The inner/outer surfaces of nanosized particles and thin films differ by an obvious topological feature. For a flat surface, the normal direction is mutually parallel at each local point, whereas the local coordinate varies for a curved spherical surface. A detailed comparison between the resonant properties of thinfilms and nanosized particles in the presence of surface anisotropy can be found in reference [75]. In this work, the surface anisotropy constants were chosen within the range of reported values for iron $\left|K_{S_{2}}\right|=\left|K_{S_{1}}\right|=$ $4 \times 10^{-4} \mathrm{~J} / \mathrm{m}^{2}$.

The dependence of $\mu_{1,2}$ on shell thickness is shown in Fig. 1(a) for different values of $K_{s}$. In the absence of surface anisotropy, the eigenvalues $k=1, n=1,2,3$ decrease with increasing $R_{1} / R_{2}$ (see. Fig. 1(a), Fig. 1(b)). This behaviour is modified for non-zero $K_{s}$, such that the eigenvaluescan either increase or decreasewith increasing $R_{1} / R_{2}$. In Fig. $1(\mathrm{a})$, the $\mu_{1,2}$ moderapidlytends to 0 with decreasing shell thickness (see Fig. 1(a)) when $K_{s}$ is opposing the resonance.The situation is different for $k=2$ eigenvalues (see Fig. 1(b)).Here, surface anisotropy plays a less significant role in determining the dependence of the eigenvalues on shell thickness, because the eigenvalue equation (15) overwhelms the surface contributioneven for large values of $K_{s}$.

In Fig. 1(b), several of the eigenvalues $\mu_{k, n}$ are plotted as a function of the ratio $R_{1} / R_{2}$. The first eigenvalue $\mu_{1,0}$ is degenerate with the ferromagnetic resonance $\left(\mu_{1,0}=0\right)$ unless surface 
anisotropy is present (see Fig. 1(b)). This eigenvalue is independent of the shell thickness for $K_{S}=0$ but has an approximately linear dependence on $R_{1} / R_{2}$ in the range $R_{1} / R_{2}=0-$ 0.6(see Fig.(b)) for non-zero $K_{S_{1}}$. In addition to shifting the frequency, surface anisotropy can deviate the magnetisation away from a homogenous single domain distribution [26]. In Fig. 1(b), the first $n=0$ mode can be expected to gradually separate from the ferromagnetic resonance as the deviation from the single domain becomes more pronounced with decreasing shell thickness.

\section{Size Dependence}

(a)

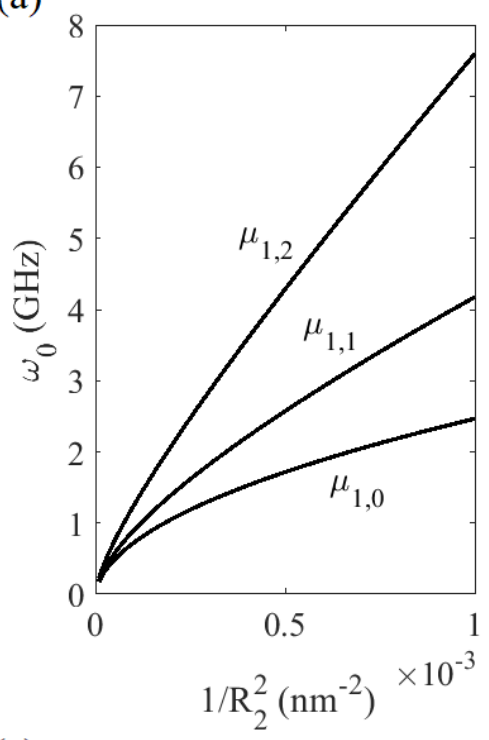

(c)

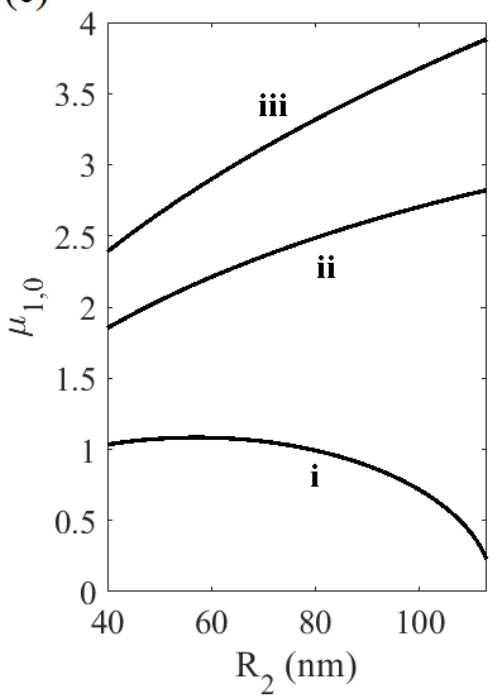

(b)

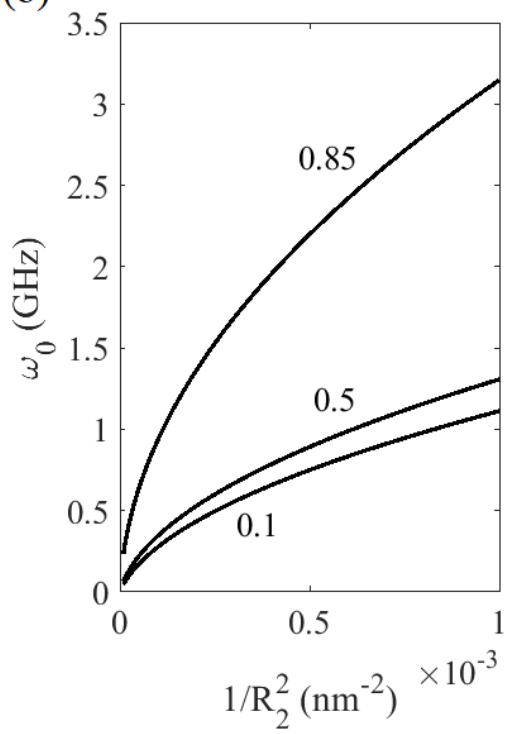

(d)

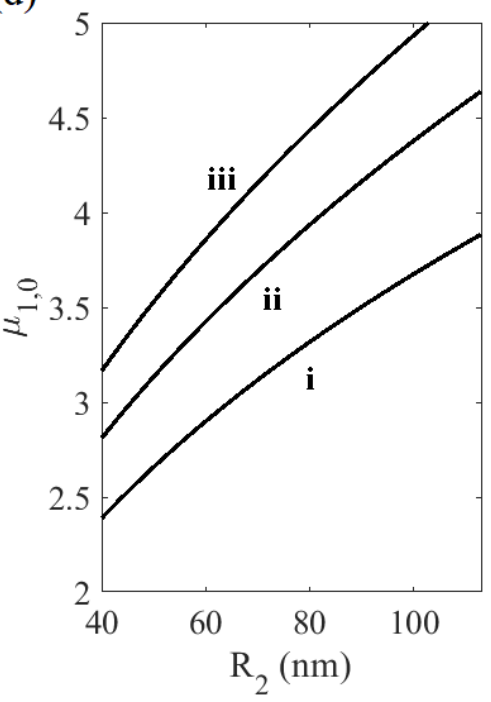

Figure 2: (a) $\mu_{1, n}$ plotted against $1 / R_{2}^{2}$ for $K_{S_{2}}=4 \times 10^{-4} \mathrm{~J} / \mathrm{m}^{2}, K_{S_{1}}=0$ and $R_{1} / R_{2}=$ 0.8 , where $\omega_{0}=\gamma_{0} C \mu_{1, n} / R_{2}^{2} M_{S}$, (b) $\mu_{1,0}$ plotted against $1 / R_{2}^{2}$ for $K_{S_{2}}=4 \times 10^{-4} \mathrm{~J} / \mathrm{m}^{2}, K_{S_{1}}=0$ and different values of $R_{1} / R_{2}$, (c) $\mu_{1,0}$ plotted against $R_{2}$ for $R_{1} / R_{2}=0.85, K_{S_{2}}=4 \times 10^{-4} \mathrm{~J} / \mathrm{m}^{2}$ and varying values of $K_{S_{1}}(i)-4 \times 10^{-4} \mathrm{~J} / \mathrm{m}^{2},(i i)-2 \times 10^{-4} \mathrm{~J} / \mathrm{m}^{2}$ and (iii) $0 \mathrm{~J} / \mathrm{m}^{2}$ and (d) The eigenvalue $\mu_{1,0}$ plotted against the outer radius $R_{2}$ for $R_{1} / R_{2}=0.85, K_{S_{2}}=4 \times 10^{-4} \mathrm{~J} / \mathrm{m}^{2}$ and varying values of $K_{s_{1}}(i) 0 \mathrm{~J} / \mathrm{m}^{2},(i i) 2 \times 10^{-4} \mathrm{~J} / \mathrm{m}^{2}$ and $(i i i) 4 \times 10^{-4} \mathrm{~J} / \mathrm{m}^{2}$. 
The eigenvalues have no dependence on $R_{2}$ when surface anisotropy is absent. A dependence on $R_{2}$ is introduced when the constants $K_{S_{2}}$ and $K_{s_{1}}$ are non-zero, such that the resonance frequencies are no longer strictly proportional to $1 / R_{2}^{2}$. The deviation from the $1 / R_{2}^{2}$ size dependence is shown in (see Fig. 2(a),(b)) when surface anisotropy is present only at the outer boundary, which could correspond to the case when a coating is applied on the outer surface. In this situation, the dependence of the eigenvalues on $R_{2}$ decreases with decreasing particle size (see Fig. 2(d)). Although the shift in the eigenvalues is decreasing with decreasing $R_{2}$, the shift in the frequency is greatly increasing due to the $1 / R_{2}^{2}$ denominator in the expression for the frequency. However, the competition between different forms of surface anisotropy at each boundary can lead to more complex effects. In Fig. 2(c), the surface anisotropy has a small impact on the eigenvalue $\mu_{1,0}$ for $R_{2}=115 \mathrm{~nm}\left(\mu_{1,0} \sim 0\right)$, but the dependence on $R_{2}$ becomes more pronounced with decreasing particle size. This is in contrast to the solid sphere [47][50] for which the dependence of the eigenvalues on $R_{2}$ always decreases with decreasing particle size.

In Fig. 2(b), the size dependence of the lowest exchange mode is shown for different values of the shell thickness. The size dependence of $\mu_{1,0}$ is close to that of the solid sphere for $R_{1} / R_{2}=$ 0.5 in Fig. 2(b), but shows large deviation from the solid sphere for $R_{1} / R_{2}=0.85$. In contrast to all other exchange modes, this mode has no size dependence in the absence of surface anisotropy. However, in the presence of surface anisotropy, the size dependence of this mode becomes pronounced as the shell thickness is decreased (see Fig. 2(b)), particularly when supported by surface contributions at the inner boundary (see Fig. 2(d)). As a result, the $\mu_{1,0}$ mode can potentially reach high frequencies in spherical shells due to the possibility of tuning the thickness.

\section{Conclusions}

The eigenvalues of the exchange resonance modes were derived for the spherical shell when assuming that surface anisotropy is present at both the inner and outer boundaries. The presence of surface anisotropy was found to play an important role in the dynamical properties of saturated nanoshells, and resulted in a range of different behaviors for lower-order $(k=1)$ eigenvalues. Relatively small values of $K_{s}$ can rapidly drive these eigenvalues towards 0 with decreasing shell thickness, suggesting that surface anisotropy is an important factor to consider in the design of high frequency microwave devices which utilize spherical shells. For higherorder modes $(k=2)$ surface anisotropy was found to play a more marginal role in determining the variation of the eigenvalues. The presence of surface anisotropy was foun ta dependence of the first $n=0$ mode on $R_{1} / R_{2}$ which led to a gradual increase in the eigenvalue with decreasing shell thickness. For this mode, similar size-dependent behavior to the solid sphere was observed up to a thickness of $R_{1} / R_{2} \sim 0.5$ when surface anisotropy was present only on the outer boundary. However, substantial deviation from the size dependence of the solid sphere was observed as the shell thickness was decreased further.

\section{ACKNOWLEDGMENTS}

The authors acknowledge financial support from the Defense Science and Technology Laboratory(DSTL) and the Engineering and Physical Sciences Research Council (EPSRC) of the United Kingdom, via the EPSRC Centre for Doctoral Training in Electromagnetic Metamaterials (Grant No. EP/L015331/1). 
[1] Sander D, Valenzuela S O, Makarov D, Marrows C H, Fullerton E E, Fischer P, McCord J, Vavassori P, Mangin S, Pirro P, Hillebrands B, Kent A D, Jungwirth T, Gutfleisch O, Kim C G and Berger A 2017 The 2017 Magnetism Roadmap J. Phys. Appl. Phys.50 363001

[2] Kim S-K, Lee K-S, Yu Y-S and Choi Y-S 2008 Reliable low-power control of ultrafast vortex-core switching with the selectivity in an array of vortex states by in-plane circular-rotational magnetic fields and spin-polarized currents Appl. Phys. Lett.92 022509

[3] Streubel R, Fischer P, Kronast F, Kravchuk V P, Sheka D D, Gaididei Y, Schmidt O G and Makarov D 2016 Magnetism in curved geometries J. Phys. Appl. Phys.49 363001

[4] Pribiag V S, Krivorotov I N, Fuchs G D, Braganca P M, Ozatay O, Sankey J C, Ralph D C and Buhrman R A 2007 Magnetic vortex oscillator driven by d.c. spinpolarized current Nat. Phys.3 498-503

[5] Ruotolo A, Cros V, Georges B, Dussaux A, Grollier J, Deranlot C, Guillemet R, Bouzehouane K, Fusil S and Fert A 2009 Phase-locking of magnetic vortices mediated by antivortices Nat. Nanotechnol.4 528-32

[6] Kim D-H, Rozhkova E A, Ulasov I V, Bader S D, Rajh T, Lesniak M S and Novosad V 2010 Biofunctionalized magnetic-vortex microdiscs for targeted cancer-cell destruction Nat. Mater.9 165-71

[7] Ferrari M 2005 Cancer nanotechnology: opportunities and challenges Nat. Rev. Cancer5 161-71

[8] Fernández-Pacheco A, Streubel R, Fruchart O, Hertel R, Fischer P and Cowburn R P 2017 Three-dimensional nanomagnetism Nat. Commun.8 15756

[9] Yin Y 2004 Formation of Hollow Nanocrystals Through the Nanoscale Kirkendall Effect Science304 711-4

[10] Buriak J M 2004 CHEMISTRY: Chemistry with Nanoscale Perfection Science304 $692-3$

[11] Son D H 2004 Cation Exchange Reactions in Ionic Nanocrystals Science306 1009-12

[12] Caruso F 1998 Nanoengineering of Inorganic and Hybrid Hollow Spheres by Colloidal Templating Science 282 1111-4

[13] Sobal N S, Hilgendorff M, Möhwald H, Giersig M, Spasova M, Radetic T and Farle M 2002 Synthesis and Structure of Colloidal Bimetallic Nanocrystals: The Non-Alloying System Ag/Co Nano Lett.2 621-4 
[14] Liu Q, Liu H, Han M, Zhu J, Liang Y, Xu Z and Song Y 2005 Nanometer-Sized Nickel Hollow Spheres Adv. Mater.17 1995-9

[15] Guo L, Liang F, Wen X, Yang S, He L, Zheng W, Chen C and Zhong Q 2007 Uniform Magnetic Chains of Hollow Cobalt Mesospheres from One-Pot Synthesis and Their Assembly in Solution Adv. Funct. Mater.17 425-30

[16] Tamion A, Raufast C, Hillenkamp M, Bonet E, Jouanguy J, Canut B, Bernstein E, Boisron O, Wernsdorfer W and Dupuis V 2010 Magnetic anisotropy of embedded Co nanoparticles: Influence of the surrounding matrix Phys. Rev. B81

[17] Jamet M, Négrier M, Dupuis V, Tuaillon-Combes J, Mélinon P, Pérez A, Wernsdorfer W, Barbara B and Baguenard B 2001 Interface magnetic anisotropy in cobalt clusters embedded in a platinum matrix J. Magn. Magn. Mater.237 293301

[18] Jamet M, Wernsdorfer W, Thirion C, Dupuis V, Mélinon P, Pérez A and Mailly D 2004 Magnetic anisotropy in single clusters Phys. Rev. B69

[19] Dorfbauer F, Evans R, Kirschner M, Chubykalo-Fesenko O, Chantrell R and Schrefl T 2007 Effects of surface anisotropy on the energy barrier in cobalt-silver core-shell nanoparticles J. Magn. Magn. Mater.316 e791-4

[20] Luis F, Bartolomé F, Petroff F, Bartolomé J, García L M, Deranlot C, Jaffrès H, Martínez M J, Bencok P, Wilhelm F, Rogalev A and Brookes N B 2006 Tuning the magnetic anisotropy of Co nanoparticles by metal capping Europhys. Lett. EPL76 142-8

[21] Yanes R, Chubykalo-Fesenko O, Evans R F L and Chantrell R W 2010 Temperature dependence of the effective anisotropies in magnetic nanoparticles with Néel surface anisotropy J. Phys. Appl. Phys.43 474009

[22] Jamet M, Wernsdorfer W, Thirion C, Mailly D, Dupuis V, Mélinon P and Pérez A 2001 Magnetic Anisotropy of a Single Cobalt Nanocluster Phys. Rev. Lett.86 4676-9

[23] Salazar-Alvarez G, Qin J, Šepelák V, Bergmann I, Vasilakaki M, Trohidou K N, Ardisson J D, Macedo W A A, Mikhaylova M, Muhammed M, Baró M D and Nogués J 2008 Cubic versus Spherical Magnetic Nanoparticles: The Role of Surface Anisotropy J. Am. Chem. Soc.130 13234-9

[24] Crespo P, Litrán R, Rojas T C, Multigner M, de la Fuente J M, Sánchez-López J C, García M A, Hernando A, Penadés S and Fernández A 2004 Permanent Magnetism, Magnetic Anisotropy, and Hysteresis of Thiol-Capped Gold Nanoparticles Phys. Rev. Lett.93

[25] Mikhaylovskiy R V, Hendry E and Kruglyak V V 2010 Negative permeability due to exchange spin-wave resonances in thin magnetic films with surface pinning Phys. Rev. B82 
[26] Usov N A and Grebenshchikov Y B 2008 Influence of surface anisotropy on magnetization distribution in a single-domain particle J. Appl. Phys.104 043903

[27] Leonov A A, Dragunov I E and Bogdanov A N 2007 Surface-induced anisotropy and multiple states in elongated magnetic nanoparticles Appl. Phys. Lett.90 193112

[28] Labaye Y, Crisan O, Berger L, Greneche J M and Coey J M D 2002 Surface anisotropy in ferromagnetic nanoparticles J. Appl. Phys.91 8715

[29] Chappert C and Bruno P 1988 Magnetic anisotropy in metallic ultrathin films and related experiments on cobalt films (invited) J. Appl. Phys.64 5736-41

[30] Lee J-W, Jeong J-R, Shin S-C, Kim J and Kim S-K 2002 Spin-reorientation transitions in ultrathin Co films on $\mathrm{Pt}(111)$ and $\mathrm{Pd}(111)$ single-crystal substrates Phys. Rev. B66

[31] Speckmann M, Oepen H P and Ibach H 1995 Magnetic Domain Structures in Ultrathin $\mathrm{Co} / \mathrm{Au}(111)$ : On the Influence of Film Morphology Phys. Rev. Lett.75 2035-8

[32] Farle M, Mirwald-Schulz B, Anisimov A N, Platow W and Baberschke K 1997 Higher-order magnetic anisotropies and the nature of the spin-reorientation transition in face-centered-tetragonal Ni(001)/Cu(001) Phys. Rev. B55 3708-15

[33] Ha K and O'Handley R C 2000 Magnetization canting in epitaxial $\mathrm{Cu} / \mathrm{Ni} / \mathrm{Cu} / \mathrm{Si}(001)$ films J. Appl. Phys. 87 5944-6

[34] Hille M, Frauen A, Beyersdorff B, Kobs A, Heße S, Frömter R and Oepen H P 2013 Direct method for measuring the canting angle of magnetization J. Appl. Phys. 113023902

[35] Gaididei Y, Kravchuk V P and Sheka D D 2014 Curvature Effects in Thin Magnetic Shells Phys. Rev. Lett.112

[36] Kravchuk V P, Rößler U K, Volkov O M, Sheka D D, van den Brink J, Makarov D, Fuchs H, Fangohr H and Gaididei Y 2016 Topologically stable magnetization states on a spherical shell: Curvature-stabilized skyrmions Phys. Rev. B94

[37] Kravchuk V P, Sheka D D, Streubel R, Makarov D, Schmidt O G and Gaididei Y 2012 Out-of-surface vortices in spherical shells Phys. Rev. B85

[38] Yan M, Kákay A, Gliga S and Hertel R 2010 Beating the Walker Limit with Massless Domain Walls in Cylindrical Nanowires Phys. Rev. Lett.104

[39] Hertel R 2013 CURVATURE-INDUCED MAGNETOCHIRALITY SPIN03 1340009

[40] Pylypovskyi O V, Kravchuk V P, Sheka D D, Makarov D, Schmidt O G and Gaididei Y 2015 Coupling of Chiralities in Spin and Physical Spaces: The Möbius Ring as a Case Study Phys. Rev. Lett.114 
[41] Vojkovic S, Carvalho-Santos V L, Fonseca J M and Nunez A S 2017 Vortexantivortex pairs induced by curvature in toroidal nanomagnets J. Appl. Phys.121 113906

[42] Carvalho-Santos V L, Moura-Melo W A and Pereira A R 2010 Miniaturization of vortex-comprising system using ferromagnetic nanotori J. Appl. Phys. 108094310

[43] Vojkovic S, Nunez A S, Altbir D and Carvalho-Santos V L 2016 Magnetization ground state and reversal modes of magnetic nanotori J. Appl. Phys.120 033901

[44] Anon F. Giesen, Ph.D. thesis, University of Hamburg, Hamburg, Germany, 2009

[45] Giesen F, Podbielski J, Korn T, Steiner M, van Staa A and Grundler D 2005 Hysteresis and control of ferromagnetic resonances in rings Appl. Phys. Lett.86 112510

[46] Mercier D, Lévy J-C S, Viau G, Fiévet-Vincent F, Fiévet F, Toneguzzo P and Acher O 2000 Magnetic resonance in spherical Co-Ni and Fe-Co-Ni particles Phys. Rev. B62 532-44

[47] Aharoni A 1997 Effect of surface anisotropy on the exchange resonance modes $J$. Appl. Phys.81 830-3

[48] Viau G, Fiévet-Vincent F, Fiévet F, Toneguzzo P, Ravel F and Acher O 1997 Size dependence of microwave permeability of spherical ferromagnetic particles $J$. Appl. Phys.81 2749-54

[49] Toneguzzo P, Viau G, Acher O, Fiévet-Vincent F and Fiévet F 1998 Monodisperse Ferromagnetic Particles for Microwave Applications Adv. Mater.10 $1032-5$

[50] Ramprecht J and Sjöberg D 2008 Magnetic losses in composite materials J. Phys. Appl. Phys.41 135005

[51] Wang H, Dai Y, Gong W, Geng D, Ma S, Li D, Liu W and Zhang Z 2013 Broadband microwave absorption of CoNi@C nanocapsules enhanced by dual dielectric relaxation and multiple magnetic resonances Appl. Phys. Lett.102 223113

[52] Ma F, Qin Y and Li Y-Z 2010 Enhanced microwave performance of cobalt nanoflakes with strong shape anisotropy Appl. Phys. Lett.96 202507

[53] Wen F, Yi H, Qiao L, Zheng H, Zhou D and Li F 2008 Analyses on double resonance behavior in microwave magnetic permeability of multiwalled carbon nanotube composites containing Ni catalyst Appl. Phys. Lett.92 042507

[54] Zhang Q, Li C, Chen Y, Han Z, Wang H, Wang Z, Geng D, Liu W and Zhang Z 2010 Effect of metal grain size on multiple microwave resonances of $\mathrm{Fe} / \mathrm{TiO} 2$ metal-semiconductor composite Appl. Phys. Lett.97 133115 
[55] Liu T, Zhou P H, Xie J L and Deng L J 2012 Electromagnetic and absorption properties of urchinlike Ni composites at microwave frequencies J. Appl. Phys.111 093905

[56] Aharoni A 1991 Exchange resonance modes in a ferromagnetic sphere J. Appl. Phys.69 7762-4

[57] Walker L R 1957 Magnetostatic Modes in Ferromagnetic Resonance Phys. Rev. 105 390-9

[58] Liu Q, Cao Q, Bi H, Liang C, Yuan K, She W, Yang Y and Che R 2016 CoNi@SiO $2 @ \mathrm{TiO}_{2}$ and CoNi@Air@TiO 2 Microspheres with Strong Wideband Microwave Absorption Adv. Mater.28 486-90

[59] Wan L, Zhang J, Chen Y, Wang H, Hu W, Liu L and Deng Y 2015 Preparation, characterization and microwave absorbing properties of nano-sized yolk-in-shell Ni-P nanospheres J. Phys. Appl. Phys.48 355302

[60] Zou J, Wang Z, Yan M and Bi H 2014 Enhanced interfacial polarization relaxation effect on microwave absorption properties of submicron-sized hollow $\mathrm{Fe}_{3} \mathrm{O}_{4}$ hemispheres J. Phys. Appl. Phys.47 275001

[61] Xu H-L, Bi H and Yang R-B 2012 Enhanced microwave absorption property of bowl-like $\mathrm{Fe}_{3} \mathrm{O}_{4}$ hollow spheres/reduced graphene oxide composites $J$. Appl. Phys.111 07A522

[62] Mu G, Chen N, Pan X, Yang K and Gu M 2007 Microwave absorption properties of hollow microsphere/titania/M-type Ba ferrite nanocomposites Appl. Phys. Lett.91 043110

[63] Yong Y, Yang Y, Wen X and Jun D 2014 Microwave electromagnetic and absorption properties of magnetite hollow nanostructures J. Appl. Phys.115 17A521

[64] Deng Y, Zhao L, Shen B, Liu L and Hu W 2006 Microwave characterization of submicrometer-sized nickel hollow sphere composites J. Appl. Phys.100 014304

[65] Zhou B, Wang Y, Li F, Tang L, Wang T and Qiao L 2017 Submicron carbonyl iron particles as an efficient microwave absorber in the low frequency band $J$. Phys. Appl. Phys.50 475001

[66] Néel L 1954 Anisotropie magnétique superficielle et surstructures d'orientation $J$. Phys. Radium15 225-39

[67] Brown, Jr. W F 1963 Micromagnetics (Wiley, New York)

[68] Aharoni A 1987 Surface anisotropy in micromagnetics J. Appl. Phys.61 3302-4

[69] Aharoni A 2002 Exchange Resonance Modes in a Hollow Sphere Phys. Status Solidi B231 547-53 
[70] Kittel C 1948 On the Theory of Ferromagnetic Resonance Absorption Phys. Rev. 73 155-61

[71] Du-Xing Chen J P-C 2016 Demagnetizing factor for a hollow sphere IEEE Magn. Lett. 7

[72] Kong D, Wang S and Chen C 2008 Magnetization ground states and phase diagrams for a nanosized Co hollow sphere: An onion-type magnetization state $J$. Appl. Phys.104 013923

[73] Burgos E, Sallica Leva E, Gómez J, Martínez Tabares F, Vásquez Mansilla M and Butera A 2011 Surface pinning in ferromagnetic films with perpendicular anisotropy Phys. Rev. B83

[74] Heinrich B, Celinski Z, Cochran J F, Arrott A S and Myrtle K 1991 Magnetic anisotropies in single and multilayered structures (invited) J. Appl. Phys.70 576974

[75] Fiorani D 2006 Surface effects in magnetic nanoparticles, Ch. 5. (Dordrecht: Springer-Verlag New York Inc.) 